\title{
Differentiation and maintenance of long-lived plasma cells
}

Kohei Kometani ${ }^{1 *}$ and Tomohiro Kurosaki ${ }^{1,2}$

${ }^{1}$ Laboratory for Lymphocyte Differentiation, RIKEN Center for Integrative Medical

Sciences (IMS), Tsurumi-ku, Yokohama, Kanagawa 230-0045, Japan

${ }^{2}$ Laboratory of Lymphocyte Differentiation, WPI Immunology Frontier Research Center,

and Graduate School of Frontier Biosciences, Osaka University, 3-1 Yamada-oka, Suita,

Osaka 565-0871, Japan

Address correspondence to Tomohiro Kurosaki. Phone: 81-6-6879-4456. E-mail:

kurosaki@ifrec.osaka-u.ac.jp

* Present address: Department of Cellular and Molecular Immunology, Max Planck

Institute of Immunobiology and Epigenetics, Freiburg, 79108, Germany 


\begin{abstract}
Long-lived plasma cells, which mostly reside in the bone marrow have been shown to be vital for protection from recurrent infections. Recent gene-targeting and cell-ablation experiments have solidified the concept that their survival depends on both cell-intrinsic programs and extrinsic factors. The BTB transcription factor ZBTB20, which is expressed at high levels in long-lived plasma cells, is critical for their survival. On the other hand, eosinophils, megakaryocytes and monocytes function to establish a niche for long-lived plasma cells by secreting cytokines and growth factors such as APRIL and IL6.
\end{abstract}




\section{Main Text}

\section{Introduction}

Long-lived plasma cells secreting high-affinity antibodies (Abs) play a very important role as a first line of defense against reinfection. Thus, elucidating the mechanisms by which long-lived plasma cells are generated and maintained is of fundamental interest to understand the basis of humoral memory.

The vast majority of long-lived plasma cells are derived from the germinal center (GC) reaction. GC is composed of two distinct regions. One is dark zone (DZ) and the other is light zone (LZ). The DZ GC B cells contain aggressively dividing cells called centroblasts and undergo somatic hyper mutations. The LZ GC B cells are $\underline{\text { non-proliferative centrocytes and are localized together with follicular dendritic cells }}$ (FDC) and follicular helper $\mathrm{T}\left(\mathrm{T}_{\mathrm{FH}}\right)$ cells. Light zone GC B cells assume one of the following three fates: plasmablasts (precursors of long-lived plasma cells), memory B cells or re-entering the GC to undergo further rounds of somatic mutation and affinity maturation (Figure). The available data on the fate decisions of light zone GC B cells suggest that, whereas very high-affinity cells have an increased probability of being directed to the plasmablast fate, lower-affinity cells can become either memory B cells or recycling GC cells. The current model for these affinity-dependent decisions postulates that light zone GC B cell clones that receive a strong enough BCR signal would then receive $\mathrm{T}$ cell help, thereby differentiating into plasmablasts[1].

Once plasmablasts are generated, they migrate through the bloodstream to find a survival niche (predominantly located in the bone marrow), where they receive survival signals, becoming long-lived plasma cells. This niche concept has been well supported by recent cellular ablation studies of megakaryocytes, eosinophils, and monocytes 
$\left[2,3_{-}^{*}, 4\right]$. In addition to the importance of such B cell extrinsic factors, B cell intrinsic programing has been also shown to contribute to survival of long-lived plasma cells $\left[5_{-}^{*}, 6_{-}^{*}\right]$. The purpose of this review is to summarize recent major advances in elucidating the cellular and molecular basis for generation and maintenance of long-lived plasma cells, with a particular focus on bone marrow plasma cells

\section{Plasmablast differentiation within the GC}

Biochemical analysis of GC B cells has been difficult because isolated ex vivo GC B cells rapidly die in vitro. Thus, the question of what signaling events in GC B cells affect their differentiation towards plasmablasts has mainly been addressed using mice deficient in candidate molecules in a GC-dependent manner.

Erk1 and Erk2 are widely expressed MAP kinase family members with many distinct signaling functions. Mice with a conditional deletion of Erk1 and Erk2 in GC B cells had relatively normal GC B cell numbers but they could not differentiate into plasmablasts [7]. Two possible mechanisms for this Erk activity have been proposed based on in vitro analyses. First, Erks are shown to be required for CD40- and cytokine-mediated induction of Blimp1, a transcriptional repressor critical for plasma cell differentiation, through phosphorylation of Elk-1 (direct model)[7]. Second, Erks contribute to induction of Blimp-1 by down-regulating transcription factors, IRF8, Bach2, and Pax5[8,9]. Bach2 and Pax5 are known to inhibit Blimp-1 expression, directly and indirectly, respectively, and indeed, down-regulation of these transcription factors takes place in the transitional stage between activated B cells and plasma cells[10-14]. Similarly, the PU.1/IRF8 complex has been recently shown to inhibit Blimp-1 expression; IRF4 binds to the Blimp-1 (Prdm1) locus in an activation-dependent manner, 
whereas PU.1/IRF8 binds to the IRF4 binding site on Prdm lin a competitive manner, thereby counteracting the IRF4 effect [15]. Apart from this transcription factor-network, post-transcriptional regulation via miRNAs may be also involved[16]. PU.1 is one of the critical targets of miR-155, thus the higher expression of miR-155 in GC B cells has been thought to facilitate plasmablast differentiation through down-regulating PU.1 mRNA translation [17-22]. This model has now been substantiated by mutagenesis of the miR-155 target site in the PU.1 3' UTR [23*].

RelA, c-Rel, and p50 are subunits of the canonical NF-אB signaling pathway, where the major heterodimers are RelA/p50 and c-Rel/p50. The in vivo role of c-Rel and RelA in GC biology has also been examined by the conditional deletion approach. c-Rel deficiency leads to the collapse of established GC[24], whereas ablation of Rel-A is dispensable for GC maintenance but essential for the development of GC-derived plasmablasts through regulation of Blimp-1 expression[24]. Thus, the canonical NF- $\kappa B$ pathway is involved in both survival of GC B cells and their differentiation into plasmablasts.

Collectively, the above data support the idea that both quantity and quality of signaling in GC B cells affect the IRF4-Blimp1 axis, thereby controlling initiation of plasmablast differentiation. But, how these signals are generated, for instance through the BCR and/or by $\mathrm{T}$ cell help, remains to be addressed.

\section{Plasmablast trafficking}

Once the antigens are conveyed to lymph node and plasmablasts are generated in the GCs, they leave the lymph nodes, enter the efferent lymphatics, circulate in the blood, and reach the bone marrow[25]. Recent data suggest the existence of several 
regulatory checkpoints operative during trafficking of plasmablasts from GCs to the bone marrow. Plasmablasts proliferate, express MHC class II and are migratory[26]. Migration has been thought to be the key for the plasmablasts to reach the places where they can receive the survival factors for their long lives; the migratory phase lasts about one week, which is a short time window within which to find a survival niche. Down-regulation of CXCR5 or CCR7[26-28] together with up-regulation of CXCR4, $\underline{E B I} 2$ and, if generated in the presence of interferon- $\gamma$, CXCR 3 allow the migration of plasmablasts out of the GCs[29-32]. After leaving GCs, plasmablasts undergo random walks within the follicle and $\mathrm{T}$ zone, reaching the medullary cords in the case of lymph nodes, where plasmablasts are retained and differentiate one step further[33**]. Myeloid cells also colonize the medullary cord and express IL6 and $\underline{\mathrm{a}}$ proliferation-inducing ligand (APRIL) mRNAs[34], suggesting that interactions between plasmablasts and myeloid cells might contribute to such differentiation. The homing of plasmablasts to the bone marrow is critical for their long-term survival and becoming $\underline{\text { long-lived plasma cells. }}$ In sphingosine-1-phosphate receptor1 (S1P1) and $\underline{\beta 2 \text {-integrin-deficient mice, plasmablasts still accumulate in the medullary cords but are }}$ unable to exit the lymph nodes and thus there is no accumulation in the bone marrow [35,36]. Concentration of S1P is high in lymph and low in the parenchyma of secondary lymphoid organs, providing a gradient used for the cell to egress, while ICAM-1 is highly expressed in medullary cords. The precise nature of the barrier between the medullary cords and the efferent lymphatics is unknown but, analogous to lymphocyte entry into lymph nodes via high endothelial venules, trans-endothelial migration is probably a central process in lymphocyte egress, possibly requiring ICAM-1 or VCAM-1-dependent processes (Figure; Step1). $\quad$ CD37 is essential for $\alpha 4 \beta 1$ integrin 
activity. Among defects observed in CD37-deficient mice (for instance GC B cell survival) one appears to be similar to that observed in $\beta 2$-integrin-deficient mice, namely accumulation of plasma cells in the spleen and absence of long-lived plasma cells from the bone marrow[37].

Because bone marrow is not connected to the lymphatic system, plasmablasts move to the bone marrow through the blood vessels and then the sinusoidal endothelial cells, which constitute the lining of sinusoids, arterioles and venules in the bone marrow, and are involved in their entry (Figure; Step2). However, the molecular mechanisms by which these endothelial cells are involved in plasmablast entry into the bone marrow remain to be determined.

\section{Niches in the bone marrow}

As plasma cells require chemokine receptor CXCR4 for their homing and/or retention in the bone marrow, it is likely that plasma cells associate with cells expressing CXCL12 - the ligand of CXCR4; indeed, almost all of the $\mathrm{IgG}^{+}$plasma cells in the bone marrow were shown to be in contact with processes or bodies of CXCL12 $2^{\text {hi }}$ reticular cells[38*]. Thus, these two cell interactions had been thought to be important for plasma cell survival. However, recent data suggest that multiple cell types collaborate to maintain the survival niche for plasma cells in the bone marrow. The initial clue was an in vivo demonstration that APRIL and its receptor BCMA play key roles in maintaining plasma cell survival in the bone marrow[39,40]. Because APRIL is poorly expressed by the CXCL12 ${ }^{\text {hi }}$ reticular cells, it was reasonable to postulate the existence of other types of cells expressing APRIL for maintenance of plasma cells in the bone marrow. A survey of various cells in the bone marrow unexpectedly revealed that the highest APRIL 
producers were the resident eosinophils[3*]. This was revealed by analysis of the following two genetically modified mice. One was the mouse strain lacking high affinity GATA-binding site in GATA-1 promoter region. Another genetically modified mouse strain expressed diphtheria toxin A under the control of eosinophil peroxidase promoter. Indeed, these two mice strains deficient in eosinophils have greatly reduced APRIL expression in the bone marrow as well as a significant reduction of bone marrow plasma cells[3*]. In regard to mechanistic insights, the observation that eosinophils express the CXCL12 receptor, CXCR4, could be important[3*,41], evoking a model in which close proximity of plasma cells to CXCL12 $2^{\text {hi }}$ reticular cells is necessary, but not sufficient. Simultaneously, eosinophils would be recruited to CXCL12 $2^{\text {hi }}$ reticular cells, thereby bringing plasma cells and eosinophils into proximity (Figure). In addition to eosinophils, megakaryocytes and monocytes also express APRIL and CXCR4, and thus can participate in the formation of bone marrow plasma cell niches[2,4].

Although the importance of APRIL is clear, other receptor-ligand interactions have been shown to be critical for survival of long-lived plasma cells. CD28, well known as a co-stimulatory molecule on T cells, is expressed on all plasma cells. CD28 deficiency in the B cell compartment only affects the maintenance of plasma cell in the bone marrow. The threshold for CD28-dependent NF- $\kappa \mathrm{B}$ activation is much lower in bone marrow plasma cells compared to splenic plasma cells[42]. Because CD80 (a ligand for CD28) is expressed on CXCL12 $2^{\text {hi }}$ reticular cells, bone marrow plasma cells might obtain significant survival advantage by their interaction with the CXCL12 $2^{\text {hi }}$ reticular cells.

\section{B cell intrinsic program for long-lived plasma cells}


What determines whether a plasmablast gives rise to a long-lived plasma cell or not? Based on the above discussion, extrinsic signals such as APRIL provided by the microenvironment are key to affect the eventual fate. However, the recent identification of B cell intrinsic transcription factors for survival of long-lived plasma cells suggests the importance of preprogramed potentiation, and this appears to be a prerequisite for receiving the extrinsic signals in the right place at the right time. In this section, our discussion will be focused on B cell intrinsic transcription factors.

Inducible deletion of Blimp-1 results in significantly reduced numbers of plasma cells in the bone marrow[43]. Because the highest levels of Blimp-1 expression are observed in the mature plasma cells in the bone marrow, the level of Blimp-1 probably contributes to the survival competence of plasma cells. However, how this maximum level is induced, and what are the targets of Blimp-1 in terms of survival of long-lived plasma cells are still unclear.

The BTB-POZ transcription factor ZBTB20, which is expressed in GC B cells and at high levels in long-lived plasma cells, is crucial for durable antibody responses $\left[5_{-}^{*}, 6_{-}^{*}\right]$. The initial events following immunization have been well analyzed in ZBTB20 knockout mice, demonstrating that loss of ZBTB20 does not affect $\mathrm{GC}$ reactions or generation of plasmablasts in the spleen[6*]. Therefore, the defect could be in trafficking to the bone marrow and/or survival of long-lived plasma cells once they reach the bone marrow. The following two lines of the evidence suggest the latter possibility. First, expression of BCMA, one of the APRIL receptors, and Mcl1 was decreased in knock-out plasma cells; indeed the importance of BCMA and Mcl1 was shown by targeting their respective genes[40,44]. Second, overexpression of anti-apoptotic factor, $\mathrm{Bcl} 2$, can rescue at least to some extent, $\mathrm{Ag}$-specific IgG responses. 
ZBTB20 turned out to be a transcriptional target of IRF4[5*]. Considering that IRF4 is up-regulated in a small subset of the GC B cells in a CD40-dependent manner, it seems possible that a ZBTB20-dependent survival program is initiated at this stage through interaction between GC B cells and follicular helper $\mathrm{T}\left(\mathrm{T}_{\mathrm{FH}}\right)$ cells. If so, then longevity of the long-lived plasma cells appears to be already imprinted at the GC stage. ZBTB20 is an obvious candidate to counteract Bcl6 activity. Because Bcl6 is essential for maintaining GCs, the anti-Bc16 effect of ZBTB20 may explain the previous observations that in vivo CD40 stimulation increases the magnitude of plasmablast differentiation while curtailing the GC reaction[45-49]. Interestingly, the requirement for ZBTB20 is dependent on the adjuvant being used; alum, but not a TLR-ligand based adjuvant, requires ZBTB20[6*]. One possibility is that the TLR-ligand based adjuvant might use similar molecule ZBTB32, instead of ZBTB20.

\section{Conclusion}

The widely accepted niche model is that besides expressing adhesion molecules and chemokines, the niche cells secrete cytokines, which are required for not only recruitment but also survival of long-lived plasma cells. In addition, recent data has highlighted the importance of B cell intrinsic program; B cell transcription factors have an also important role for determining the fates for long-lived plasma cells.

$\underline{\text { In regard to the interactions between niche cells and plasma cells, }}$ cytokines/chemokines produced by niche cells have been so far well appreciated. However, given the recent evidence that plasma cells and plasmablasts themselves secrete stimulatory (IL-17, GM-CSF, TNF $\alpha$ ) as well as inhibitory cytokines (IL-10, IL-35) [50-55], another regulatory mechanisms are also worth considering. The niche 
activation and survival could be regulated by these plasma cell-derived cytokines. Thus, it is reasonable to imagine that mutual interdependent loops between plasma cells and niche cells could be established, thereby creating a more dynamic regulation of the process. 


\section{Acknowledgements}

We thank Dr. K. Suzuki and Dr. P. D. Burrows for critical reading of our manuscript. This work was supported by grants provided by the Ministry of Education, Culture, Sports, Science, and Technology in Japan (to T.K.); Japan Science and Technology Agency, Core Research for Evolutional Science and Technology (CREST) (to T.K.); Secom Science and Technology Foundation (to T.K.); RIKEN Special Postdoctoral Researchers Program (to K.K.). 


\section{References and recommended reading}

* of special interest

** of outstanding interest

1. Victora GD, Nussenzweig MC: Germinal centers. Annu. Rev. Immunol. 2012, 30:429-457.

2. Winter O, Moser K, Mohr E, Zotos D, Kaminski H, Szyska M, Roth K, Wong DM, Dame C, Tarlinton DM, et al.: Megakaryocytes constitute a functional component of a plasma cell niche in the bone marrow. Blood 2010, 116:1867-1875.

3. Chu VT, Fröhlich A, Steinhauser G, Scheel T, Roch T, Fillatreau S, Lee JJ, LOhning M, Berek C: Eosinophils are required for the maintenance of plasma cells in the bone marrow. Nat. Immunol. 2011, 12:151-159.

** This paper reported eosinophil provide a survival niche for long-lived plasma cells by secreting APRIL and IL6.

4. Belnoue E, Tougne C, Rochat A-F, Lambert P-H, Pinschewer DD, Siegrist C-A: Homing and adhesion patterns determine the cellular composition of the bone marrow plasma cell niche. J. Immunol. 2012, 188:1283-1291.

5. Chevrier S, Emslie D, Shi W, Kratina T, Wellard C, Karnowski A, Erikci E, Smyth GK, Chowdhury K, Tarlinton D, et al.: The BTB-ZF transcription factor Zbtb20 is driven by Irf4 to promote plasma cell differentiation and longevity. J. Exp. Med. 2014, 211:827-840. 
* Refs [5, 6] identified ZBTB20 is essential for the maintenance of long-live plasma cells.

6. Wang Y, Bhattacharya D: Adjuvant-specific regulation of long-term antibody responses by ZBTB20. J. Exp. Med. 2014, 211:841-856.

* Refs [5, 6] identified ZBTB20 is essential for the maintenance of long-live plasma cells.

7. Yasuda T, Kometani K, Takahashi N, Imai Y, Aiba Y, Kurosaki T: ERKs induce expression of the transcriptional repressor Blimp-1 and subsequent plasma cell differentiation. Sci Signal 2011, 4:ra25.

8. Le Gallou S, Caron G, Delaloy C, Rossille D, Tarte K, Fest T: IL-2 requirement for human plasma cell generation: coupling differentiation and proliferation by enhancing MAPK-ERK signaling. J. Immunol. 2012, 189:161-173.

9. Yasuda T, Hayakawa F, Kurahashi S, Sugimoto K, Minami Y, Tomita A, Naoe T: B cell receptor-ERK1/2 signal cancels PAX5-dependent repression of BLIMP1 through PAX5 phosphorylation: a mechanism of antigen-triggering plasma cell differentiation. J. Immunol. 2012, 188:6127-6134.

10. Ochiai K, Katoh Y, Ikura T, Hoshikawa Y, Noda T, Karasuyama H, Tashiro S, Muto A, Igarashi K: Plasmacytic transcription factor Blimp-1 is repressed by Bach2 in B cells. J. Biol. Chem. 2006, 281:38226-38234.

11. Delogu A, Schebesta A, Sun Q, Aschenbrenner K, Perlot T, Busslinger M: Gene repression by Pax5 in B cells is essential for blood cell homeostasis and is reversed in plasma cells. Immunity 2006, 24:269-281. 
12. Nera K-P, Kohonen P, Narvi E, Peippo A, Mustonen L, Terho P, Koskela K, Buerstedde J-M, Lassila O: Loss of Pax5 promotes plasma cell differentiation. Immunity 2006, 24:283-293.

13. Kallies A, Hasbold J, Fairfax K, Pridans C, Emslie D, McKenzie BS, Lew AM, Corcoran LM, Hodgkin PD, Tarlinton DM, et al.: Initiation of plasma-cell differentiation is independent of the transcription factor Blimp-1. Immunity 2007, 26:555-566.

14. Muto A, Ochiai K, Kimura Y, Itoh-Nakadai A, Calame KL, Ikebe D, Tashiro S, Igarashi K: Bach2 represses plasma cell gene regulatory network in B cells to promote antibody class switch. EMBO 2010, 29:4048-4061.

15. Carotta S, Willis SN, Hasbold J, Inouye M, Pang SHM, Emslie D, Light A, Chopin M, Shi W, Wang H, et al.: The transcription factors IRF8 and PU.1 negatively regulate plasma cell differentiation. J. Exp. Med. 2014, 211:2169-2181.

16. O'Connell RM, Rao DS, Chaudhuri AA, Baltimore D: Physiological and pathological roles for microRNAs in the immune system. Nat. Rev. Immunol. 2010, 10:111-122.

17. Rodriguez A, Vigorito E, Clare S, Warren MV, Couttet P, Soond DR, van Dongen S, Grocock RJ, Das PP, Miska EA, et al.: Requirement of bic/microRNA-155 for normal immune function. Science 2007, 316:608-611. 
18. Thai T-H, Calado DP, Casola S, Ansel KM, Xiao C, Xue Y, Murphy A, Frendewey D, Valenzuela D, Kutok JL, et al.: Regulation of the germinal center response by microRNA-155. Science 2007, 316:604-608.

19. Vigorito E, Perks KL, Abreu-Goodger C, Bunting S, Xiang Z, Kohlhaas S, Das PP, Miska EA, Rodriguez A, Bradley A, et al.: microRNA-155 regulates the generation of immunoglobulin class-switched plasma cells. Immunity 2007, 27:847-859.

20. Teng G, Hakimpour P, Landgraf P, Rice A, Tuschl T, Casellas R, Papavasiliou FN: MicroRNA-155 is a negative regulator of activation-induced cytidine deaminase. Immunity 2008, 28:621-629.

21. Dorsett Y, McBride KM, Jankovic M, Gazumyan A, Thai T-H, Robbiani DF, Di Virgilio M, Reina San-Martin B, Heidkamp G, Schwickert TA, et al.:

MicroRNA-155 suppresses activation-induced cytidine deaminase-mediated Myc-Igh translocation. Immunity 2008, 28:630-638.

22. Kuchen S, Resch W, Yamane A, Kuo N, Li Z, Chakraborty T, Wei L, Laurence A, Yasuda T, Peng S, et al.: Regulation of microRNA expression and abundance during lymphopoiesis. Immunity 2010, 32:828-839.

23. Lu D, Nakagawa R, Lazzaro S, Staudacher P, Abreu-Goodger C, Henley T, Boiani S, Leyland R, Galloway A, Andrews S, et al.: The miR-155-PU.1 axis acts on Pax5 to enable efficient terminal B cell differentiation. J. Exp. Med. 2014, 211:2183-2198. 
* This study showed miR-155 targets PU.1 3' UTR and regulates plasma cell differentiation.

24. Heise N, De Silva NS, Silva K, Carette A, Simonetti G, Pasparakis M, Klein U: Germinal center B cell maintenance and differentiation are controlled by distinct NF-кB transcription factor subunits. J. Exp. Med. 2014, 211:2103-2118.

25. Radbruch A, Muehlinghaus G, Luger EO, Inamine A, Smith KGC, Dörner T, Hiepe F: Competence and competition: the challenge of becoming a long-lived plasma cell. Nat. Rev. Immunol. 2006, 6:741-750.

26. Kallies A, Hasbold J, Tarlinton DM, Dietrich W, Corcoran LM, Hodgkin PD, Nutt SL: Plasma cell ontogeny defined by quantitative changes in blimp-1 expression. J. Exp. Med. 2004, 200:967-977.

27. Wehrli N, Legler DF, Finke D, Toellner KM, Loetscher P, Baggiolini M, MacLennan IC, Acha-Orbea $\mathrm{H}$ : Changing responsiveness to chemokines allows medullary plasmablasts to leave lymph nodes. Eur. J. Immunol. 2001, 31:609-616.

28. Hargreaves DC, Hyman PL, Lu TT, Ngo VN, Bidgol A, Suzuki G, Zou YR, Littman DR, Cyster JG: A coordinated change in chemokine responsiveness guides plasma cell movements. J. Exp. Med. 2001, 194:45-56.

29. Muehlinghaus G, Cigliano L, Huehn S, Peddinghaus A, Leyendeckers H, Hauser AE, Hiepe F, Radbruch A, Arce S, Manz RA: Regulation of CXCR3 and 
CXCR4 expression during terminal differentiation of memory B cells into plasma cells. Blood 2005, 105:3965-3971.

30. Gatto D, Paus D, Basten A, Mackay CR, Brink R: Guidance of B Cells by the Orphan G Protein-Coupled Receptor EBI2 Shapes Humoral Immune Responses. Immunity 2009, 31:259-269.

31. Pereira JP, Kelly LM, Xu Y, Cyster JG: EBI2 mediates B cell segregation between the outer and centre follicle. Nature 2009, 460:1122-1126.

32. Hauser AE, Debes GF, Arce S, Cassese G, Hamann A, Radbruch A, Manz RA: Chemotactic responsiveness toward ligands for CXCR3 and CXCR4 is regulated on plasma blasts during the time course of a memory immune response. J. Immunol. 2002, 169:1277-1282.

33. Fooksman DR, Schwickert TA, Victora GD, Dustin ML, Nussenzweig MC, Skokos D: Development and migration of plasma cells in the mouse lymph node. Immunity 2010, 33:118-127.

** This paper delineated the pre-plasma cell migration using intravital microscopy

34. Mohr E, Serre K, Manz RA, Cunningham AF, Khan M, Hardie DL, Bird R, MacLennan ICM: Dendritic cells and monocyte/macrophages that create the IL-6/APRIL-rich lymph node microenvironments where plasmablasts mature. J. Immunol. 2009, 182:2113-2123. 
35. Kabashima K, Haynes NM, Xu Y, Nutt SL, Allende ML, Proia RL, Cyster JG: Plasma cell S1P1 expression determines secondary lymphoid organ retention versus bone marrow tropism. $J$. Exp. Med. 2006, 203:2683-2690.

36. Pabst O, Peters T, Czeloth N, Bernhardt G, Scharffetter-Kochanek K, Förster R: Cutting edge: egress of newly generated plasma cells from peripheral lymph nodes depends on beta 2 integrin. $J$. Immunol. 2005, 174:7492-7495.

37. van Spriel AB, de Keijzer S, van der Schaaf A, Gartlan KH, Sofi M, Light A, Linssen PC, Boezeman JB, Zuidscherwoude M, Reinieren-Beeren I, et al.: The tetraspanin CD37 orchestrates the $\alpha(4) \beta(1)$ integrin-Akt signaling axis and supports long-lived plasma cell survival. Sci Signal 2012, 5:ra82.

38. Tokoyoda K, Egawa T, Sugiyama T, Choi B-I, Nagasawa T: Cellular niches controlling B lymphocyte behavior within bone marrow during development. Immunity 2004, 20:707-718.

* This paper showed $\mathrm{IgG}^{+}$plasma cell are associated with CXLC12 ${ }^{\text {hi }}$ reticular cell in bone marrow.

39. Benson MJ, Dillon SR, Castigli E, Geha RS, Xu S, Lam K-P, Noelle RJ: Cutting edge: the dependence of plasma cells and independence of memory $B$ cells on BAFF and APRIL. J. Immunol. 2008, 180:3655-3659.

40. O'Connor BP, Raman VS, Erickson LD, Cook WJ, Weaver LK, Ahonen C, Lin L-L, Mantchev GT, Bram RJ, Noelle RJ: BCMA is essential for the survival of long-lived bone marrow plasma cells. J. Exp. Med. 2004, 199:91-98. 
41. Nagase H, Miyamasu M, Yamaguchi M, Fujisawa T, Ohta K, Yamamoto K, Morita Y, Hirai K: Expression of CXCR4 in eosinophils: functional analyses and cytokine-mediated regulation. J. Immunol. 2000, 164:5935-5943.

42. Rozanski CH, Arens R, Carlson LM, Nair J, Boise LH, Chanan-Khan AA, Schoenberger SP, Lee KP: Sustained antibody responses depend on CD28 function in bone marrow-resident plasma cells. $J$. Exp. Med. 2011, 208:1435-1446.

43. Shapiro-Shelef M, Lin K-I, Savitsky D, Liao J, Calame K: Blimp-1 is required for maintenance of long-lived plasma cells in the bone marrow. J. Exp. Med. 2005, 202:1471-1476.

44. Peperzak V, Vikstrom I, Walker J, Glaser SP, Lepage M, Coquery CM, Erickson LD, Fairfax K, Mackay F, Strasser A, et al.: Mcl-1 is essential for the survival of plasma cells. Nat. Immunol. 2013, 14:290-297.

45. Uchida J, Yasui T, Takaoka-Shichijo Y, Muraoka M, Kulwichit W, Raab-Traub N, Kikutani H: Mimicry of CD40 signals by Epstein-Barr virus LMP1 in B lymphocyte responses. Science 1999, 286:300-303.

46. Erickson LD, Durell BG, Vogel LA, O'Connor BP, Cascalho M, Yasui T, Kikutani H, Noelle RJ: Short-circuiting long-lived humoral immunity by the heightened engagement of CD40. J. Clin. Invest. 2002, 109:613-620.

47. Hömig-Hölzel C, Hojer C, Rastelli J, Casola S, Strobl LJ, Müller W, Quintanilla-Martinez L, Gewies A, Ruland J, Rajewsky K, et al.: Constitutive 
CD40 signaling in B cells selectively activates the noncanonical NF-kappaB pathway and promotes lymphomagenesis. J. Exp. Med. 2008, 205:1317-1329.

48. Bolduc A, Long E, Stapler D, Cascalho M, Tsubata T, Koni PA, Shimoda M: Constitutive CD40L expression on B cells prematurely terminates germinal center response and leads to augmented plasma cell production in $\mathrm{T}$ cell areas. J. Immunol. 2010, 185:220-230.

49. Kishi Y, Aiba Y, Higuchi T, Furukawa K, Tokuhisa T, Takemori T, Tsubata T: Augmented antibody response with premature germinal center regression in CD40L transgenic mice. J. Immunol. 2010, 185:211-219.

50. Bermejo DA, Jackson SW, Gorosito-Serran M, Acosta-Rodriguez EV, Amezcua-Vesely MC, Sather BD, Singh AK, Khim S, Mucci J, Liggitt D, et al.: Trypanosoma cruzi trans-sialidase initiates a program independent of the transcription factors ROR $\gamma \mathrm{t}$ and Ahr that leads to IL-17 production by activated B cells. Nat. Immunol. 2013, 14:514-522.

51. Rauch PJ, Chudnovskiy A, Robbins CS, Weber GF, Etzrodt M, Hilgendorf I, Tiglao E, Figueiredo J-L, Iwamoto Y, Theurl I, et al.: Innate response activator B cells protect against microbial sepsis. Science 2012, 335:597-601.

52. Fritz JH, Rojas OL, Simard N, McCarthy DD, Hapfelmeier S, Rubino S, Robertson SJ, Larijani M, Gosselin J, Ivanov II, et al.: Acquisition of a multifunctional IgA+ plasma cell phenotype in the gut. Nature 2012, 481:199-203. 
53. Neves P, Lampropoulou V, Calderon-Gomez E, Roch T, Stervbo U, Shen P, Kühl AA, Loddenkemper C, Haury M, Nedospasov SA, et al.: Signaling via the MyD88 adaptor protein in B cells suppresses protective immunity during Salmonella typhimurium infection. Immunity 2010, 33:777-790.

54. Shen P, Roch T, Lampropoulou V, O'Connor RA, Stervbo U, Hilgenberg E, Ries S, Dang VD, Jaimes Y, Daridon C, et al.: IL-35-producing B cells are critical regulators of immunity during autoimmune and infectious diseases. Nature 2014, 507:366-370.

55. Matsumoto M, Baba A, Yokota T, Nishikawa H, Ohkawa Y, Kayama H, Kallies A, Nutt SL, Sakaguchi S, Takeda K, et al.: Interleukin-10-producing plasmablasts exert regulatory function in autoimmune inflammation. Immunity $2014 \mathrm{Dec}$ $\underline{18 ; 41(6)}$ 


\section{Figure legend}

Plasmablasts migrate to bone marrow and become long-lived plasma cells. In the lymph node, B cells differentiate to short-lived plasmablasts residing in extrafollicular or germinal center (GC) B cells. High affinity light zone (LZ) GC B cells (centrocytes) have a high propensity to differentiate to plasmablasts (1). Low affinity LZ GC B cells are thought to give rise to memory B cells (2) or entering re-GC reaction by migrating into dark zone (DZ) (3). GC-derived plasmablasts downregulate CXCR5 and leave GC. These plasmablasts move to medullary sinus and enter efferent lymphatic vessel (Step1). After moving in lymphatic system, plasmablasts go through blood vessel and migrate to the bone marrow. Medullary vasucular sinuses in the bone marrow are surrounded by endothelial cells. Plasmablasts enter bone marrow probably by transmigrating through endothelial cells (Step2). In the bone marrow, CXCL12-expressing reticular cells recruit CXCR4-expressing plasma cells, eosinophils, and monocytes. Eosinophils, Megakaryocytes, and Monocytes secrete APRIL and IL6. Plasmablasts which receive these survival factors differentiate into long-lived plasma cells and maintain continuous antibody production. 


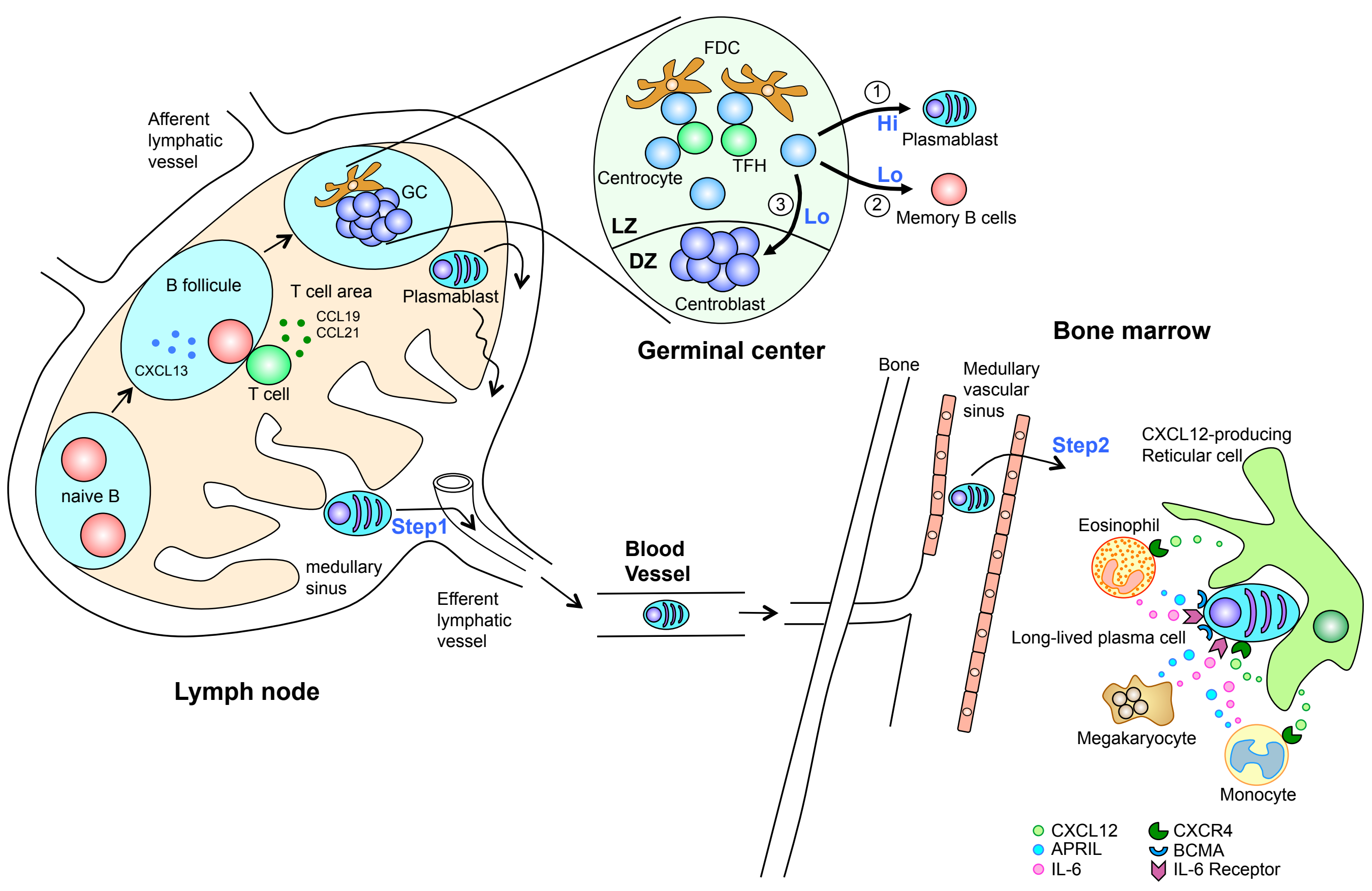

